\title{
Degree distribution in random planar graphs
}

\author{
Michael Drmota ${ }^{1}$ and Omer Giménez ${ }^{2}$ and Marc Noy $\|^{2}$ \\ ${ }^{1}$ Tecnische Universität Wien, Wiedner Haupstrasse 8-10, A-1040 Wien, Austria. \\ ${ }^{2}$ Universitat Politècnica de Catalunya, Jordi Girona 1-3, 08034 Barcelona, Spain.
}

\begin{abstract}
We prove that for each $k \geq 0$, the probability that a root vertex in a random planar graph has degree $k$ tends to a computable constant $d_{k}$, and moreover that $\sum_{k} d_{k}=1$. The proof uses the tools developed by Giménez and Noy in their solution to the problem of the asymptotic enumeration of planar graphs, and is based on a detailed analysis of the generating functions involved in counting planar graphs. However, in order to keep track of the degree of the root, new technical difficulties arise. We obtain explicit, although quite involved expressions, for the coefficients in the singular expansions of interest, which allow us to use transfer theorems in order to get an explicit expression for the probability generating function $p(w)=\sum_{k} d_{k} w^{k}$. From the explicit expression for $p(w)$ we can compute the $d_{k}$ to any degree of accuracy, and derive asymptotic estimates for large values of $k$.
\end{abstract}

Keywords: Planar graph, Random graph, Degree distribution.

\section{Introduction}

In this paper all graphs are simple and labelled with labels $\{1,2, \ldots, n\}$. As usual, a graph is planar if it can be embedded in the plane without edge crossings. A planar graph together with a particular embedding in the plane is called a map. There is a rich theory of counting maps, and part of it is needed later on. However, in this paper we consider planar graphs as combinatorial objects, regardless of how many non-equivalent topological embeddings they may have.

Random planar graphs were introduced by Denise et al. (7), and since then they have been widely studied. Let us first recall the probability model. Let $\mathcal{G}_{n}$ be the family of (labelled) planar graphs with $n$ vertices. A random planar graph $\mathcal{R}_{n}$ is a graph drawn from $\mathcal{G}_{n}$ with the uniform distribution, that is, all planar graphs with $n$ vertices have the same probability of being chosen. As opposed to the classical Erdős-Rényi model, we cannot produce a random planar graph by drawing edges independently. In fact, our analysis of random planar graphs relies on exact and asymptotic counting.

Several natural parameters defined on $\mathcal{R}_{n}$ have been studied, starting with the number of edges, which is probably the most basic one. Partial results where obtained in (7, 12, 21, 5), until it was shown by Giménez and Noy (13) that the number of edges in random planar graphs obeys asymptotically a normal limit law with linear expectation and variance. The expectation is asymptotically $\kappa n$, where $\kappa \approx 2.21326$ is a well-defined analytic constant. This implies that the average degree of the vertices is $2 \kappa \approx 4.42652$.

\footnotetext{
${ }^{\dagger}$ Research supported in part by Project MTM2005-08618C02-01. 
McDiarmid et al. (18) showed that with high probability a planar graph has a linear number of vertices of degree $k$, for each $k \geq 1$. Our main result is that for each $k \geq 1$, the expected number of vertices of degree $k$ is asymptotically $d_{k} n$, for computable constants $d_{k} \geq 0$. This is equivalent to saying that the probability that a fixed vertex, say vertex 1 , has degree $k$ tends to a limit $d_{k}$ as $n$ goes to infinity. In Theorem 6.9 we show that this limit exists and we give an explicit expression for the probability generating function

$$
p(w)=\sum_{k \geq 1} d_{k} w^{k}
$$

from which the coefficients $d_{k}$ can be computed to any degree of accuracy. Moreover, we show that $p(w)$ is indeed a probability generating function, that is, $\sum d_{k}=1$.

The proof is based on a detailed analysis of the generating functions involved in counting planar graphs, as developed in (13), where the long standing problem of counting planar graphs asymptotically was solved. However, in this case we need to keep track of the degree of a root vertex, and this makes the analysis considerably more difficult.

Here is a sketch of the paper. After some preliminaries, we obtain the degree distribution in simpler families of planar graphs: outerplanar graphs (Section 3) and series-parallel graphs (Section 4). These results are interesting on their own and pave the way to the more complex analysis of general planar graphs. We remark that the degree distribution in these simpler cases has been obtained independently in (3) using different techniques.

In Section 5 we compute the generating function of 3-connected maps taking into account the degree of the root, which is an essential piece in proving the main result. We rely on a classical bijection between rooted maps and rooted quadrangulations (6, 19), and again the main difficulty is to keep track of the root degree. The task is completed in Section 6, which contains the analysis for planar graphs. First we have to obtain a closed form for the generating function $B^{\bullet}(x, y, w)$ of rooted 2-connected planar graphs, where $x$ marks vertices, $y$ edges, and $w$ the degree of the root: the main problem we encounter here is solving a differential equation involving algebraic functions and other functions defined implicitly. The second step is to obtain singular expansions of the various generating functions near their dominant singularities; this is particularly demanding, as the coefficients of the singular expansions are rather complex expressions. Finally, using a technical lemma on singularity analysis and composition of singular expansions, we are able to work out the asymptotics for the generating function $C^{\bullet}(x, y, w)$ of rooted connected planar graphs, and from this the probability generating function can be computed exactly. We also compute the degree distribution for 3-connected and 2-connected planar graphs, and for planar graphs according to the edge density.

For each of the three families studied we obtain an explicit expression for the probability generating function $p(w)=\sum_{k \geq 1} d_{k} w^{k}$, of increasing complexity. Theorems 3.2 4.3 and 6.9 give the exact expressions in each case. The following tables show the approximate values for small values of $k$, and the asymptotic behaviour for large $k$. It is worth noticing that the shape of the asymptotic estimates for planar graphs agrees with the general pattern for the degree distribution in several classes of maps (15), where maps are counted according to the number of edges.

McDiarmid and Reed (17) have shown that the maximum degree in random planar graphs is $\Theta(\log n)$. From the asymptotic results in Theorem 6.9 we are led to conjecture that if $\Delta_{n}$ denotes the maximum 


\begin{tabular}{|l|c|c|c|c|c|c|}
\hline & $d_{1}$ & $d_{2}$ & $d_{3}$ & $d_{4}$ & $d_{5}$ & $d_{6}$ \\
\hline Outerplanar & 0.1365937 & 0.2875331 & 0.2428739 & 0.1550795 & 0.0874382 & 0.0460030 \\
Series-Parallel & 0.1102133 & 0.3563715 & 0.2233570 & 0.1257639 & 0.0717254 & 0.0421514 \\
Planar & 0.0367284 & 0.1625794 & 0.2354360 & 0.1867737 & 0.1295023 & 0.0861805 \\
\hline
\end{tabular}

Tab. 1: Degree distribution for small degrees.

\begin{tabular}{|l|l|l|}
\hline Outerplanar & $c \cdot k^{1 / 4} e^{\sqrt{k}} q^{k}$ & $q \approx 0.3808138$ \\
Series-Parallel & $c \cdot k^{-3 / 2} q^{k}$ & $q \approx 0.7504161$ \\
Planar & $c \cdot k^{-1 / 2} q^{k}$ & $q \approx 0.6734506$ \\
\hline
\end{tabular}

Tab. 2: Asymptotic estimates of $d_{k}$ for large $k$. The constants $c$ and $q$ in each case are defined analytically.

degree in random planar graphs, then the expected value is asymptotically

$$
E \Delta_{n} \sim \frac{\log n}{\log (1 / q)}
$$

where $q$ is as in Theorem 6.9 We remark that an analogous result holds for planar maps (11), counted according to the number of edges.

Let us mention that in a companion paper (8), we prove a central limit theorem for the number of vertices of degree $k$ in outerplanar and series-parallel graphs, together with strong concentration results. It remains an open problem to show that this is also the case for planar graphs. Our results in the present paper show that the degree distribution exists and can be computed explicitly. For lack of space we omit: proofs; the coefficients of singular expansions; and the degree distribution for 2-connected graphs in each case, as well as for 3-connected planar graphs.

\section{Preliminaries}

For background on generating functions associated to planar graphs, we refer to (13) and (4), and to (20) for a less technical description. For background on singularity analysis of generating functions, we refer to the (9) and to the forthcoming book by Flajolet and Sedgewick (10).

For each class of graphs under consideration, $c_{n}$ and $b_{n}$ denote, respectively, the number of connected and 2-connected graphs on $n$ vertices. For the three graphs classes under consideration, outerplanar, series-parallel, and planar, we have both for $c_{n}$ and $b_{n}$ estimates of the form

$$
c \cdot n^{-\alpha} \rho^{-n} n !,
$$

where $c, \alpha$ and $\rho$ are suitable constants (4; 13). For outerplanar and series-parallel graphs we have $\alpha=$ $-5 / 2$, whereas for planar graphs $\alpha=-7 / 2$. A general methodology for graph enumeration explaining these critical exponents has been developed in (14). 
We introduce the exponential generating functions $C(x)=\sum c_{n} x^{n} / n$ ! and $B(x)=\sum b_{n} x^{n} / n$ !. Let $C_{k}$ be the exponential generating function (GF for short) for rooted connected graphs, where the root bears no label and has degree $k$; that is, the coefficient $\left[x^{n} / n !\right] C_{k}(x)$ equals the number of rooted connected graphs with $n+1$ vertices, in which the root has no label and has degree $k$. Analogously we define $B_{k}$ for 2-connected graphs. Also, let

$$
B^{\bullet}(x, w)=\sum B_{k}(x) w^{k}, \quad C^{\bullet}(x, w)=\sum C_{k}(x) w^{k} .
$$

Notice that $C^{\bullet}(x, 1)=C^{\prime}(x)$.

A basic property shared by the classes of outerplanar, series-parallel and planar graphs is that a connected graph $G$ is in the class if and only if the 2-connected components of $G$ are also in the class. As shown in (13), this implies the basic equation

$$
C^{\prime}(x)=e^{B^{\prime}\left(x C^{\prime}(x)\right)}
$$

between univariate GFs. If we introduce the degree of the root, then the equation becomes

$$
C^{\bullet}(x, w)=e^{B^{\bullet}\left(x C^{\prime}(x), w\right)} .
$$

The reason is that only the 2-connected components containing the root vertex contribute to its degree.

Our goal in each case is to estimate $\left[x^{n}\right] C_{k}(x)$, since the limit probability that a given fixed vertex has degree $k$ is equal to

$$
d_{k}=\lim _{n \rightarrow \infty} \frac{\left[x^{n}\right] C_{k}(x)}{\left[x^{n}\right] C^{\prime}(x)} .
$$

A first observation is that the asymptotic degree distribution is the same for connected members of a class than for all members in the class. Let $G(x)$ be the GF for all members in the class, and let $G_{k}(x)$ be the GF of all rooted graphs in the class, where the root has degree $k$. Then we have

$$
G(x)=e^{C(x)}, \quad G_{k}(x)=C_{k}(x) e^{C(x)} .
$$

The first equation is standard, and in the second equation the factor $C_{k}(x)$ corresponds to the connected component containing the root, and the second factor to the remaining components. The functions $G(x)$ and $C(x)$ have the same dominant singularity. Given the singular expansions of $G(x)$ and $C(x)$ at the dominant singularity in each of the cases under consideration, it follows that

$$
\lim _{n \rightarrow \infty} \frac{\left[x^{n}\right] G_{k}(x)}{\left[x^{n}\right] G^{\prime}(x)}=\lim _{n \rightarrow \infty} \frac{\left[x^{n}\right] C_{k}(x)}{\left[x^{n}\right] C^{\prime}(x)} .
$$

Hence, in each case we only need to determine the degree distribution for connected graphs. A more intuitive explanation is that the largest component in random planar graphs eats up almost everything: the expected number of vertices not in the largest component is constant (16).

Another observation is that $d_{0}=0$ and $d_{1}=\rho$, where $\rho$ is the constant appearing in the estimate (1) for $c_{n}$; as we are going to see, $\rho$ is the radius of convergence of $C(x)$. Indeed, there are no vertices of degree zero in a connected graph, and the proportion of rooted connected graphs in which the root has degree one is $n(n-1) c_{n-1} / n c_{n} \sim \rho$. 
The general approach we use for computing the $d_{k}$ is the following. Let $f(x)=x C^{\prime}(x)$ and let $H(z)=e^{B^{\bullet}(z, w)}$, where $w$ is considered as a parameter. Let also $\rho$ be the radius of convergence of $C(x)$, which is the same as that of $f(x)$. According to 2$)$ we have to estimate $\left[x^{n}\right] H(f(x))$, and this will depend on the behaviour of $H(z)$ at $z=f(\rho)$. In the outerplanar and series-parallel cases, $H(z)$ turns out be analytic at $f(\rho)$, whereas in the planar case we have a critical composition scheme, that is, the dominant singularity of $H(z)$ is precisely $f(\rho)$. This is a fundamental difference and we have to use different tools accordingly. Another difference is that $B^{\bullet}$ is much more difficult to determine for planar graphs.

\section{Outerplanar graphs}

We start by recalling some results from (4). From the equivalence between rooted 2-connected outerplanar graphs and polygon dissections where the vertices are labelled $1,2, \ldots, n$ in clockwise order (see Section 5 in (4) for details), we have the explicit expression

$$
B^{\prime}(x)=\frac{1+5 x-\sqrt{1-6 x+x^{2}}}{8} .
$$

The radius of convergence of $B(x)$ is $3-2 \sqrt{2}$, the smallest positive root of $1-6 x+x^{2}=0$. The radius of convergence of $C(x)$ is $\rho=\psi(\tau)$, where $\psi(u)=u e^{-B^{\prime}(u)}$, and $\tau$ is the unique positive root of $\psi^{\prime}(u)=0$. Notice that $\tau$ satisfies $\tau B^{\prime \prime}(\tau)=0$. The approximate values are $\tau \approx 0.17076$ and $\rho \approx 0.13659$. We also need the fact that $\psi$ is the functional inverse of $x C^{\prime}(x)$, so that $\tau=\rho C^{\prime}(\rho)$.

Let

$$
D(x)=\frac{1+x-\sqrt{1-6 x+x^{2}}}{4}
$$

and let $D_{k}(x)=x(2 D(x)-x)^{k-1}\left(D_{k}\right.$ is the ordinary GF for polygon dissections in which the root vertex has degree $k$ ). Then we have

$$
B_{k}=\frac{1}{2} D_{k}, k \geq 2, \quad B_{1}=x .
$$

By summing a geometric series we have an explicit expression for $B^{\bullet}$, namely

$$
B^{\bullet}(x, w)=x w+\sum_{k=2}^{\infty} \frac{x}{2}(2 D(x)-x)^{k-1} w^{k}=x w+\frac{x w^{2}}{2} \frac{2 D(x)-x}{1-(2 D(x)-x) w} .
$$

Our goal is to analyze $B^{\bullet}(x, w)$ and $C^{\bullet}(x, w)=\exp \left(B^{\bullet}\left(x C^{\prime}(x), w\right)\right)$. For this we need the following technical lemma.

Lemma 3.1 Let $f(x)=\sum_{n \geq 0} a_{n} x^{n} / n$ ! denote the exponential generating function of a sequence $a_{n}$ of non-negative real numbers and assume that $f(x)$ has exactly one dominating square-root singularity at $x=\rho$ of the form

$$
f(x)=g(x)-h(x) \sqrt{1-x / \rho},
$$

where $g(x)$ and $h(x)$ are analytic at $x=\rho$ and $f(x)$ has an analytic continuation to the region $\{x \in \mathbb{C}$ : $|x|<\rho+\varepsilon\} \backslash\{x \in \mathbb{R}: x \geq \rho\}$ for some $\varepsilon>0$. Further, let $H(x, z)$ denote a function that is analytic for $|x|<\rho+\varepsilon$ and $|z|<f(\rho)+\varepsilon$ such that $H_{z}(\rho, f(\rho)) \neq 0$. Then the function

$$
f_{H}(x)=H(x, f(x))
$$


has a power series expansion $f_{H}(x)=\sum_{n \geq 0} b_{n} x^{n} / n$ ! and the coefficients $b_{n}$ satisfy

$$
\lim _{n \rightarrow \infty} \frac{b_{n}}{a_{n}}=H_{z}(\rho, f(\rho))
$$

Applying the previous lemma with $f(x)=B^{\prime}(x)$ and $H(x, z)=x w+\frac{x w^{2}}{2} \frac{4 z-3 x}{1-(4 z-3 x) w}$, we obtain the following.

Theorem 3.2 Let $d_{k}$ be the limit probability that a vertex of a connected outerplanar graph has degree $k$. then

$$
p(w)=\sum_{k \geq 1} d_{k} w^{k}=\left.\rho \cdot \frac{\partial}{\partial x} e^{B^{\bullet}(x, w)}\right|_{x=\rho C^{\prime}(\rho)},
$$

where $B^{\bullet}$ is given by Equations (4) and (5).

Moreover $p(1)=1$, so that the $d_{k}$ are indeed a probability distribution and we have asymptotically, as $k \rightarrow \infty$

$$
d_{k} \sim c_{1} k^{1 / 4} e^{c_{2} \sqrt{k}} q^{k}
$$

where $c_{1}, c_{2}$ are suitable constants, and $q=2 D(\tau)-\tau \approx 0.3808138$.

\section{Series-parallel graphs}

First we recall the necessary results from (4). The radius of convergence of $C(x)$ is, as for outerplanar graphs, $\rho=\psi(\tau)$, where $\psi(u)=u e^{-B^{\prime}(u)}$, and $\tau$ is the unique positive root of $\psi^{\prime}(u)=0$. Again we have that $\psi$ is the functional inverse of $x C^{\prime}(x)$, so that $\tau=\rho C^{\prime}(\rho)$, and $\tau$ satisfies $\tau B^{\prime \prime}(\tau)=0$. The approximate values are $\tau \approx 0.12796$ and $\rho \approx 0.11021$.

In order to study 2-connected series-parallel graphs, we need to consider series-parallel networks, as in (4). We recall that a network is a graph with two distinguished vertices, called poles, such that the multigraph obtained by adding an edge between the two poles is 2-connected. Let $D(x, y, w)$ be the exponential GF of series-parallel networks, where $x, y, w$ mark, respectively, vertices, edges, and the degree of the first pole. Define $S(x, y, w)$ analogously for series networks. Then we have

$$
\begin{aligned}
D(x, y, w) & =(1+y w) e^{S(x, y, w)}-1 \\
S(x, y, w) & =(D(x, y, w)-S(x, y, w)) x D(x, y, 1)
\end{aligned}
$$

The first equation reflects the fact that a network is a parallel composition of series networks, and the second one the fact that a series network is obtained by connecting a non-series network with an arbitrary network (see (22) for details); the factor $D(x, y, 1)$ appears because we only keep track of the degree of the first pole.

Remark. For the results of the present section, we do not need to take into account the number of edges and we could set $y=1$ everywhere. However, in the case of planar graphs we do need the GF according to all three variables and it is convenient to present already here the full development. In the proof of the main result of this section, Theorem 4.3 , we just set $y=1$. 
Set $E(x, y)=D(x, y, 1)$, the GF for series-parallel networks without marking the degree of the root, which satisfies (see (4)) the equation

$$
\log \left(\frac{1+E(x, y)}{1+y}\right)=\frac{x E(x, y)^{2}}{1+x E(x, y)}
$$

It follows that

$$
\log \left(\frac{1+D(x, y, w)}{1+y w}\right)=\frac{x E(x, y) D(x, y, w)}{1+x E(x, y)} .
$$

We also have the following relation between the $B_{k}$ and $S$.

\section{Lemma 4.1}

$$
w \frac{\partial B^{\bullet}(x, y, w)}{\partial w}=\sum_{k \geq 1} k B_{k}(x, y) w^{k}=x y w e^{S(x, y, w)} .
$$

¿From the previous equation it follows that

$$
B^{\bullet}(x, y, w)=x y \int e^{S(x, y, w)} d w
$$

Our next task is to get rid of the integral and to express $B^{\bullet}$ in terms of $D$. Recall that $E(x, y)=D(x, y, 1)$.

Lemma 4.2 The generating function of rooted 2-connected series-parallel graphs is equal to

$$
B^{\bullet}(x, y, w)=x\left(D(x, y, w)-\frac{x E(x, y)}{1+x E(x, y)} D(x, y, w)\left(1+\frac{D(x, y, w)}{2}\right)\right) .
$$

Theorem 4.3 Let $d_{k}$ be the limit probability that a vertex of a connected series-parallel graph has degree $k$. then

$$
p(w)=\sum_{k \geq 1} d_{k} w^{k}=\left.\rho \cdot \frac{\partial}{\partial x} e^{B^{\bullet}(x, 1, w)}\right|_{x=\rho C^{\prime}(\rho)}
$$

where $B^{\bullet}$ is given by Lemma 4.2 and Equations (8) and (7).

Moreover $p(1)=1$, so that the $d_{k}$ are indeed a probability distribution and we have asymptotically, as $k \rightarrow \infty$

$$
d_{k} \sim c \cdot k^{-3 / 2} q^{k}
$$

where $c$ is a positive constant and

$$
q=\left((1+1 /(\tau E(\tau, 1))) e^{-1 / \tau E(\tau, 1)}-1\right)^{-1} \approx 0.7504161
$$

\section{Quadrangulations and 3-connected planar graphs}

The goal of this section is to find the generating function of 3-connected planar graphs according to the degree of the root. This is an essential ingredient in the next section.

First we work out the problem for simple quadrangulations, which are in bijection with 3-connected maps. In order to do that we must revisit the classical work of Brown and Tutte (6) on 2-connected (nonseparable) maps. Finally, using the fact that a 3-connected planar graph has a unique embedding, we finish the job. 


\subsection{Simple quadrangulations}

A rooted quadrangulation is a planar map where every face is a quadrangle, and with a distinguished directed edge of the external face, which is called the root edge of the quadrangulation. The root vertex of the quadrangulation is the first vertex of the root edge. A diagonal is an internal path of length 2 joining two opposite vertices of the external face. A quadrangulation is simple if it has no diagonal, every cycle of length 4 other than the external one defines a face, and it is not the trivial map reduced to a single quadrangle. In Section 5 of (19) it is shown how to count simple quadrangulations. Here we extend this result to count them also according to the degree of the root vertex.

A quadrangulation is bipartite and connected, so if we fix the colour of the root vertex there is a unique way of 2-colouring the vertices. We call the two colours black and white, and we assume the root is black. Diagonals are called black or white, according to the colour of the external vertices they join.

Let $F(x, y, w)$ be the GF of rooted quadrangulations, where the variables $x, y$ and $w$ mark, respectively, the number of black vertices minus one, the number of white vertices minus one, and the degree of the root vertex minus one. Generating functions for maps are always ordinary, since maps are unlabelled objects.

The generating functions $F_{N}, F_{B}$ and $F_{W}$ are associated, respectively, to quadrangulations with no diagonal, to those with at least one black diagonal (at the root vertex), and to those with at least one whit diagonal (not at the root vertex). By planarity only one of the two kinds of diagonals can appear in a quadrangulation; it follows that

$$
F(x, y, w)=F_{N}(x, y, w)+F_{B}(x, y, w)+F_{W}(x, y, w) .
$$

A quadrangulation with a diagonal can be decomposed into two quadrangulations, by considering the maps to the left and to the right of this diagonal. This gives raise to the equations

$$
\begin{aligned}
& F_{B}(x, y, w)=\left(F_{N}(x, y, w)+F_{W}(x, y, w)\right) \frac{F(x, y, w)}{x}, \\
& F_{W}(x, y, w)=\left(F_{N}(x, y, w)+F_{B}(x, y, w)\right) \frac{F(x, y, 1)}{y} .
\end{aligned}
$$

In the second case, only one of the two quadrangulations contribute to the degree of the root vertex; this is the reason why the term $F(x, y, 1)$ appears. The $x$ and the $y$ in the denominators appear because the three vertices of the diagonal are common to the two quadrangulations. Since we are considering vertices minus one, we only need to correct the colour that appears twice at the diagonal. Incidentally, no term $w$ appears in the equations for the same reason.

Let us write $F=F(x, y, w)$ and $F(1)=F(x, y, 1)$. From the previous equations we deduce that

$$
\begin{aligned}
& F=F_{N}+F_{B}+F_{W}=\left(F_{N}+F_{B}\right)\left(1+\frac{F}{x}\right), \\
& F=F_{N}+F_{B}+F_{W}=\left(F_{N}+F_{W}\right)\left(1+\frac{F(1)}{y}\right),
\end{aligned}
$$

so that

$$
F+F_{N}=\left(F_{N}+F_{B}\right)+\left(F_{N}+F_{W}\right)=F\left(\frac{1}{1+\frac{F}{x}}+\frac{1}{1+\frac{F(1)}{y}}\right)
$$


and finally

$$
F_{N}=F\left(\frac{1}{1+\frac{F}{x}}+\frac{1}{1+\frac{F(1)}{y}}-1\right) .
$$

Now we proceed to count simple quadrangulations. We use the following combinatorial decomposition of quadrangulations with no diagonals in terms of simple quadrangulations: all quadrangulations with no diagonals, with the only exception of the trivial one, can be decomposed uniquely into a simple quadrangulation $q$ and as many quadrangulations as internal faces $q$ has (replace every internal face of $q$ by its corresponding quadrangulations)

Let

$$
Q(x, y, w)=\sum_{i, j, k} q_{i, j, k} x^{i} y^{j} w^{k},
$$

be the GF of simple quadrangulations, where $x, y$ and $w$ have the same meaning as for $F$. [Note: In (19) this GF is called $Q_{N}^{*}$.] We translate the combinatorial decomposition of simple quadrangulations into generating functions as follows.

$$
\begin{aligned}
F_{N}(x, y, w)-x y w & =\sum_{i, j, k} q_{i, j, k} x^{i} y^{j}\left(\frac{F}{x y}\right)^{k}\left(\frac{F(1)}{x y}\right)^{i+j-1-k} \\
& =\sum_{i, j, k} q_{i, j, k} \frac{x y}{F(1)}\left(\frac{F(1)}{y}\right)^{i}\left(\frac{F(1)}{x}\right)^{j}\left(\frac{F}{F(1)}\right)^{k}= \\
& =\frac{x y}{F(1)} Q\left(\frac{F(1)}{y}, \frac{F(1)}{x}, \frac{F}{F(1)}\right),
\end{aligned}
$$

where we are using the fact that a quadrangulation counted by $q_{i, j, k}$ has $i+j+2$ vertices, $i+j-1$ internal faces, and $k$ of them are incident to the root vertex.

At this point we change variables as $X=F(1) / y, Y=F(1) / x$ and $W=F / F(1)$. Then Equations (10) and 111) can be rewritten as

$$
\begin{gathered}
\frac{x y}{F(1)} Q(X, Y, W)=F_{N}-x y w=F\left(\frac{1}{1+\frac{F}{x}}+\frac{1}{1+\frac{F(1)}{y}}-1\right)-x y w, \\
Q(X, Y, W)=X Y W\left(\frac{1}{1+W Y}+\frac{1}{1+X}-1\right)-F(1) w .
\end{gathered}
$$

The last equation would be an explicit expression of $Q$ in terms of $X, Y, W$ if it were not for the term $F(1) w=F(x, y, 1) w$. In (19) it is shown that

$$
F(1)=\frac{R S}{(1+R+S)^{3}},
$$

where $R=R(X, Y)$ and $S(X, Y)$ are algebraic functions defined by

$$
R=X(S+1)^{2}, \quad S=Y(R+1)^{2} .
$$

Hence it remains only to obtain an expression for $w=w(X, Y, W)$ to obtain an explicit expression for $Q$. This done in the next subsection. 


\subsection{Rooted non-separable planar maps}

In (6), the authors studied the generating function $h(x, y, w)$ of rooted non-separable planar maps where $x, y$ and $w$ count, respectively the number of vertices minus one, the number of faces minus one, and the valency (number of edges) of the external face. [Note: In (6) the variable $z$ is used instead of $w$.] There is a bijection between rooted quadrangulations and non-separable rooted planar maps: black and white vertices in the quadrangulation correspond, respectively, to faces and vertices of the map; quadrangles become edges; and the root vertex becomes the external face, and its degree becomes its valency). As a consequence $h(y, x, w)=w F(x, y, w)$, where the extra factor $w$ appears because in $F$ we are counting the degree of the root vertex minus one. It follows that Equation (3.9) from (6) becomes

$$
(1-w)(1-y w) w F=-w^{2} F^{2}+(-x w+w F(1)) w F+x w^{2}(x(1-w)+F(1))
$$

By dividing both sides by $F(1)^{2}$, and rewriting in terms of $X=F(1) / y, Y=F(1) / x$ and $W=F / F(1)$, we obtain

$$
\begin{gathered}
(1-w)\left(\frac{1}{F(1)}-\frac{w}{X}\right) w W=-w^{2} W^{2}+\left(1-\frac{1}{Y}\right) w^{2} W+\frac{w^{2}}{Y}\left(\frac{1}{X}(1-w)+1\right) \\
\frac{(1-w)(X-w F(1)) w W}{X F(1)}=\frac{w^{2}\left(-X Y W^{2}+X Y W-X W+1-w+X\right.}{X Y} \\
Y(1-w)(X-w F(1)) W=w F(1)\left(-X Y W^{2}+X Y W-X W+1-w+X\right) .
\end{gathered}
$$

Observe that this is a quadratic equation in $w$. Solving for $w$ in (15) and using (13) and (14) we get (the plus sign is because $T^{\bullet}$ has positive coefficients in coming Theorem 5.1

$$
w=\frac{-w_{1}(R, S, W)+(R-W+1) \sqrt{w_{2}(R, S, W)}}{2(S+1)^{2}\left(S W+R^{2}+2 R+1\right)},
$$

where $w_{1}(R, S, W)$ and $w_{2}(R, S, W)$ are polynomials given by

$$
\begin{aligned}
w_{1}= & -R S W^{2}+W\left(1+4 S+3 R S^{2}+5 S^{2}+R^{2}+2 R+2 S^{3}+3 R^{2} S+7 R S\right) \\
& +(R+1)^{2}\left(R+2 S+1+S^{2}\right) \\
w_{2}= & R^{2} S^{2} W^{2}-2 W R S\left(2 R^{2} S+6 R S+2 S^{3}+3 R S^{2}+5 S^{2}+R^{2}+2 R+4 S+1\right) \\
& +(R+1)^{2}\left(R+2 S+1+S^{2}\right)^{2}
\end{aligned}
$$

The reason we choose to write $w$ as a function of $(R, S, W)$ instead of $(X, Y, W)$ will become clear later on.

Thus, together with Equations (12) and (13), we have finally obtained an explicit expression for the generating function $Q(X, Y, W)$ of simple quadrangulations in terms of $W$ and algebraic functions $R(X, Y)$ and $S(X, Y)$. 


\subsection{3-connected planar graphs}

Let $T^{\bullet}(x, z, w)$ be the GF of 3-connected planar graphs, where one edge is taken as the root and given a direction, and where $x$ counts vertices, $z$ counts edges, and $w$ counts the degree of the tail of the root edge. Now we relate $T^{\bullet}$ to the $\mathrm{GF} Q(X, Y, W)$ of simple quadrangulations.

By the bijection between simple quadrangulations and 3-connected planar maps, and using Euler's relation, the $\mathrm{GF} x w Q(x z, z, w)$ counts rooted 3-connected planar maps, where $z$ marks edges (we have added an extra term $w$ to correct the 'minus one' in the definition of $Q$ ).

By Whitney's theorem 3-connected planar graphs have a unique embedding on the sphere. As noticed in (1), the are two ways of rooting an embedding of a directed edge-rooted graph in order to get a rooted map, since there are two ways of choosing the root face adjacent to the root edge. It follows that

$$
T^{\bullet}(x, z, w)=\frac{x w}{2} Q(x z, z, w) .
$$

Theorem 5.1 The generating function of directed edge-rooted 3-connected planar graphs, where $x, z, w$ mark, respectively, vertices, edges, and the degree of the root vertex, is equal to

$$
T^{\bullet}=\frac{x^{2} z^{2} w^{2}}{2}\left(\frac{1}{1+w z}+\frac{1}{1+x z}-1-\frac{(u+1)^{2}\left(-w_{1}(u, v, w)+(u-w+1) \sqrt{w_{2}(u, v, w)}\right)}{2 w\left(v w+u^{2}+2 u+1\right)(1+u+v)^{3}}\right)
$$

where $u$ and $v$ are algebraic functions defined by

$$
u=x z(1+v)^{2}, \quad v=z(1+u)^{2},
$$

and $w_{1}(u, v, w)$ and $w_{2}(u, v, w)$ are given by (17) and 18) replacing $R, S, W$ by $u, v, w$, respectively.

\section{Planar graphs}

\subsection{2-connected planar graphs}

Let $B^{\bullet}(x, y, w)$, the generating function of rooted 2-connected planar graphs taking into account the degree of the root. As for series series-parallel graphs we have to work with networks.

Let $T^{\bullet}(x, z, w)$ be the GF for directed edge-rooted 3-connected planar maps as in the previous section. As in Section 4 , we denote by $D(x, y, w)$ and $S(x, y, w)$, respectively, the GFs of (planar) networks and series networks, with the same meaning for the variables $x, y$ and $w$.

Lemma 6.1 We have

$$
\begin{aligned}
D(x, y, w) & =(1+y w) \exp \left(S(x, y, w)+\frac{1}{x^{2} D(x, y, w)} T^{\bullet}\left(x, E(x, y), \frac{D(x, y, w)}{E(x, y)}\right)\right)-1 \\
S(x, y, w) & =x E(x, y)(D(x, y, w)-S(x, y, w)),
\end{aligned}
$$

where $E(x, y)=D(x, y, 1)$ is the GF for planar networks (without marking the degree of the root).

As in Lemma 4.1. and for the same reason, we have

$$
w \frac{\partial B^{\bullet}(x, y, w)}{\partial w}=x y w \exp \left(S(x, y, w)+\frac{1}{x^{2} D(x, y, w)} T^{\bullet}\left(x, E(x, y), \frac{D(x, y, w)}{E(x, y)}\right)\right)
$$


Lemma 6.2 The generating function of rooted 2-connected planar graphs is equal to

$$
B^{\bullet}(x, y, w)=x\left(D-\frac{x E D}{1+x E}\left(1+\frac{D}{2}\right)\right)-\frac{1+D}{x D} T^{\bullet}(x, E, D / E)+\frac{1}{x} \int_{0}^{D} \frac{T^{\bullet}(x, E, t / E)}{t} d t,
$$

where for simplicity we let $D=D(x, y, w)$ and $E=E(x, y)$.

In order to get a full expression for $B^{\bullet}(x, y, w)$, it remains to compute the integral in the formula of the previous lemma.

Lemma 6.3 Let $T^{\bullet}(x, z, w)$ be the GF of 3-connected planar graphs as before. Then

$$
\begin{aligned}
& \int_{0}^{w} \frac{T^{\bullet}(x, z, t)}{t} d t=-\frac{x^{2}\left(z^{3} x w^{2}-2 w z-2 x z^{2} w+(2+2 x z) \log (1+w z)\right)}{4(1+x z)} \\
& -\frac{u v x}{2(1+u+v)^{3}}\left(\frac{w\left(2 u^{3}+(6 v+6) u^{2}+\left(6 v^{2}-v w+14 v+6\right) u+4 v^{3}+10 v^{2}+8 v+2\right)}{4 v(v+1)^{2}}\right. \\
& +\frac{(1+u)\left(1+u+2 v+v^{2}\right)\left(2 u^{3}+(4 v+5) u^{2}+\left(3 v^{2}+8 v+4\right) u+2 v^{3}+5 v^{2}+4 v+1\right)}{4 u v^{2}(v+1)^{2}} \\
& -\frac{\sqrt{Q}\left(2 u^{3}+(4 v+5) u^{2}+\left(3 v^{2}-v w+8 v+4\right) u+5 v^{2}+2 v^{3}+4 v+1\right)}{4 u v^{2}(v+1)^{2}} \\
& +\frac{(1+u)^{2}(1+u+v)^{3} \log \left(Q_{1}\right)}{2 v^{2}(1+v)^{2}} \\
& \left.+\frac{\left(u^{3}+2 u^{2}+u-2 v^{3}-4 v^{2}-2 v\right)(1+u+v)^{3} \log \left(Q_{2}\right)}{2 v^{2}(1+v)^{2} u}\right)
\end{aligned}
$$

where the expressions $Q, Q_{1}$ and $Q_{2}$ are given by

$$
\begin{aligned}
Q & =u^{2} v^{2} w^{2}-2 u v w\left(u^{2}(2 v+1)+u\left(3 v^{2}+6 v+2\right)+2 v^{3}+5 v^{2}+4 v+1\right) \\
& +(1+u)^{2}\left(u+(v+1)^{2}\right)^{2} \\
Q_{1} & =\frac{1}{2\left(w v+(u+1)^{2}\right)^{2}(v+1)\left(u^{2}+u(v+2)+(v+1)^{2}\right)}\left(-u v w\left(u^{2}+u(v+2)+2 v^{2}+3 v+1\right)\right. \\
& +(u+1)(u+v+1) \sqrt{Q}+(u+1)^{2}\left(2 u^{2}(v+1)+u\left(v^{2}+3 v+2\right)+v^{3}+3 v^{2}+3 v+1\right) \\
Q_{2} & =\frac{-w u v+u^{2}(2 v-1)+u\left(3 v^{2}+6 v+2\right)+2 v^{3}+5 v^{2}+4 v+1-\sqrt{Q}}{2 v\left(u^{2}+u(v+2)+(v+1)^{2}\right)}
\end{aligned}
$$

Combining Lemmas 6.2 and 6.3 we can produce an explicit (although quite long) expression for $B^{\bullet}(x, y, w)$ in terms of $D(x, y, w), E(x, y)$, and the algebraic functions $u(x, y), v(x, y)$. This is needed in the next section for computing the singular expansion of $B^{\bullet}(x, y, w)$ at its dominant singularity.

\subsection{Singular expansions}

In this section we find singular expansions of $T^{\bullet}(x, z, w), D(x, y, w)$ and $B^{\bullet}(x, y, w)$ at their dominant singularities. As we show here, these singularities do not depend on $w$ and were found in (1) and (13). 
However the coefficients of the singular expansions do depend on $w$, and our task is to compute them exactly in each case.

What is needed in the next section is the singular expansion for $B^{\bullet}$, but to compute it we first need the singular expansions of $u, v, T^{\bullet}$ and $D$ (for $u$ and $v$ compare also with (2) and (1)).

Lemma 6.4 Suppose that $x$ and $w$ are sufficiently close to the positive real axis and that $|w| \leq 1$. Then the dominant singularity $z=\tau(x)$ of $T^{\bullet}(x, z, w)$ does not depend on $w$. The singular expansion at $\tau(x)$ is

$$
T^{\bullet}(x, z, w)=T_{0}(x, w)+T_{2}(x, w) Z^{2}+T_{3}(x, w) Z^{3}+O\left(Z^{4}\right),
$$

where $Z=\sqrt{1-z / \tau(x)}$, and the expressions for the $T_{i}$ are analytic functions of $x$ and $w$.

Similary we get an alternate expansion in the variable $x$.

Lemma 6.5 Suppose that $z$ and $w$ are sufficiently close to the positive real axis and that $|w| \leq 1$. Then the dominant singularity $x=r(z)$ of $T^{\bullet}(x, z, w)$ does not depend on $w$. The singular expansion at $r(z)$ is

$$
T^{\bullet}(x, z, w)=\widetilde{T}_{0}(z, w)+\widetilde{T}_{2}(z, w) X^{2}+\widetilde{T}_{3}(x, w) X^{3}+O\left(X^{4}\right) .
$$

Using Lemma 6.1 and the previous result we obtain the following.

Lemma 6.6 Suppose that $y$ and $w$ are sufficiently close to the positive real axis and that $|w| \leq 1$. Then the dominant singularity $x=R(y)$ of $D(x, y, w)$ does not depend on $w$. The singular expansion at $R(y)$ is

$$
D(x, y, w)=D_{0}(y, w)+D_{2}(y, w) X^{2}+D_{3}(y, w) X^{3}+O\left(X^{4}\right)
$$

where $X=\sqrt{1-x / R(y)}$, and the $D_{i}$ are analytic functions.

And finally from Lemmas 6.2 and 6.3 we obtain the singular expansion for $B^{\bullet}$, which is the essential piece in our analysis.

Lemma 6.7 Suppose that $y$ and $w$ are sufficiently close to the positive real axis and that $|w| \leq 1$. Then the dominant singularity $x=R(y)$ of $B^{\bullet}(x, y, w)$ does not depend on $w$, and is the same as for $D(x, y, w)$. The singular expansion at $R(y)$ is

$$
B^{\bullet}(x, y, w)=B_{0}(y, w)+B_{2}(y, w) X^{2}+B_{3}(y, w) X^{3}+O\left(X^{4}\right)
$$

where $X=\sqrt{1-x / R(y)}$, and the $B_{i}$ are analytic functions.

\subsection{Degree distribution for planar graphs}

The following is the analogous of Lemma 3.1. The difference now is that we are composing two singular expansions and, moreover, they are of type $3 / 2$.

Lemma 6.8 Let $f(x)=\sum_{n \geq 0} a_{n} x^{n} / n$ ! denote the exponential generating function of a sequence $a_{n}$ of non-negative real numbers and suppose that $f(x)$ has exactly one dominating singularity at $x=\rho$ of the form

$$
f(x)=f_{0}+f_{2} X^{2}+f_{3} X^{3}+\mathcal{O}\left(X^{4}\right),
$$


where $X=\sqrt{1-x / \rho}$, and has an analytic continuation to the region $\{x \in \mathbb{C}:|x|<\rho+\varepsilon\} \backslash\{x \in$ $\mathbb{R}: x \geq \rho\}$ for some $\varepsilon>0$. Further, let $H(x, z, w)$ denote a function that has a dominant singularity at $z=f(\rho)>0$ of the form

$$
H(x, z, w)=h_{0}(x, w)+h_{2}(x, w) Z^{2}+h_{3}(x, w) Z^{3}+\mathcal{O}\left(Z^{4}\right),
$$

where $w$ is considered as a parameter, $Z=\sqrt{1-z / f(\rho)}$, the functions $h_{j}(x, w)$ are analytic in $x$, and $H(x, z, w)$ has an analytic continuation...

Then the function

$$
f_{H}(x)=H(x, f(x), w)
$$

has a power series expansion $f_{H}(x)=\sum_{n \geq 0} b_{n} x^{n} / n$ ! and the coefficients $b_{n}$ satisfy

$$
\lim _{n \rightarrow \infty} \frac{b_{n}}{a_{n}}=-\frac{h_{2}(\rho, w)}{f_{0}}+\frac{h_{3}(\rho, w)}{f_{3}}\left(-\frac{f_{2}}{f_{0}}\right)^{3 / 2} .
$$

Applying the previous lemma with $H(x, z, w)=x e^{B^{\bullet}(z, 1, w)}$ and $f(x)=x C^{\prime}(x)$, we obtain:

Theorem 6.9 Let $d_{k}$ be the probability that a vertex of a connected planar graph has degree $k$. Then

$$
\begin{aligned}
p(w)=\sum_{k \geq 1} d_{k} w^{k} & =-e^{B_{0}(1, w)-B_{0}(1,1)} B_{2}(1, w) \\
& +e^{B_{0}(1, w)-B_{0}(1,1)} \frac{1+B_{2}(1,1)}{B_{3}(1,1)} B_{3}(1, w),
\end{aligned}
$$

where $B_{j}(y, w), j=0,2,3$ are the analytic functions given in Lemma 6.7

Moreover, $p(1)=1$, so that the $d_{k}$ are indeed a probability distribution and we have asymptotically, as $k \rightarrow \infty$,

$$
d_{k} \sim c k^{-1 / 2} q^{k}
$$

where $c$ is a positive constant and $q \approx 0.6734506$.

\section{References}

[1] E. A. Bender, Z. Gao, N. C. Wormald, The number of 2-connected labelled planar graphs, Elec. J. Combinatorics 9 (2002), \#43.

[2] E.A. Bender, L.B. Richmond, The asymptotic enumeration of rooted convex polyhedra, J. Combin. Theory B 3 (1994) 276-283.

[3] N. Bernasconi, K. Panagiotou, A. Steger, On the degree sequences of random and outerplanar and series-parallel graphs (manuscript).

[4] M. Bodirsky, O. Giménez, M. Kang, and M. Noy, Enumeration and limit laws for series-parallel graphs, Europ. J. Combinatorics 28 (2007), 2091-2105.

[5] N. Bonichon, C. Gavoille, N. Hanusse, D. Poulalhon, G. Schaeffer, Planar Graphs, via Well-Orderly Maps and Trees, Graphs and Combinatorics 22 (2006), 185-202. 
[6] W. G. Brown, W. T. Tutte, On the enumeration of rooted non-separable planar maps, Canad. J. Math. 16 (1964) 572-577.

[7] A. Denise, M. Vasconcellos, D. J. A. Welsh, The random planar graph, Congr. Numer. 113 (1996), 61-79.

[8] M. Drmota, O. Giménez, M. Noy, The number of vertices of given degree in series-parallel graphs (submitted).

[9] P. Flajolet, A. Odlyzko, Singularity analysis of generating functions, SIAM J. Discrete Math. 3 (1990), 216-240.

[10] P. Flajolet and R. Sedgewick, Analytic combinatorics, to be published in 2008 by Cambridge University Press, preliminary version available at http://algo.inria.fr/flajolet/Publications

[11] Z. Gao, N. C. Wormald, The distribution of the maximum vertex degree in random planar maps. J. Combin. Theory Ser. A 89 (2000), 201-230.

[12] S. Gerke, C. McDiarmid, On the Number of Edges in Random Planar Graphs, Comb. Prob. and Computing 13 (2004), 165-183.

[13] O. Giménez, M. Noy, The number of planar graphs and properties of random planar graphs, DMTCS Proceedings, vol. AD, 2005, pp. 147-156. Full version to appear in J. Amer Math. Soc.

[14] O. Giménez, M. Noy, J. Rué, Graph classes with given 3-connected components: asymptotic counting and critical phenomena, Proc. of Eurcocomb 2007, Elec. Notes in Discrete Math. 29 (2007), $521-529$.

[15] V. A. Liskovets, A pattern of asymptotic vertex valency distributions in planar maps, J. Combin. Theory Ser. B 75 (1999), 116-133.

[16] C. McDiarmid, Random graphs on surfaces, J. Combin. Theory Ser. B. 98 (2008), 778-797.

[17] C. McDiarmid, B. Reed, On the maximum degree of a random planar graph (manuscript).

[18] C. McDiarmid, A. Steger, D. J. A. Welsh, Random planar graphs. J. Combin. Theory Ser. B 93 (2005), 187-205.

[19] R. C, Mullin, P. J. Schellenberg, The enumeration of $c$-nets via quadrangulations, J. Combin. Theory 4 (1968), 259-276.

[20] M. Noy, Random planar graphs and the number of planar graphs in Combinatorics, Complexity, and Chance: A Tribute to Dominic Welsh (2007), pp. 213-233.

[21] D. Osthus, H. J. Prömel, A. Taraz, On random planar graphs, the number of planar graphs and their triangulations, J. Combin. Theory Ser. B 88 (2003), 119-134.

[22] T. R. S. Walsh, Counting labelled three-connected and homeomorphically irreducible two-connected graphs, J. Combin. Theory Ser. B 32 (1982), 1-11. 
